\title{
Deployment Strategy of Wireless Sensor Networks: A Survey
}

\author{
Pei Jiang ${ }^{1,2, a}$, Li Dong², Xueliang Pang ${ }^{2}$ \\ ${ }^{1}$ Mechanical Engineering Postdoctoral Research Station, Huazhong University of Science and \\ Technology, Wuhan, 430033, China \\ ${ }^{2}$ Naval Engineering University, Wuhan, 430033, China \\ ajiangpei@vip.163.com
}

Keywords: wireless sensor networks; node deployment; sensing model; deployment strategy; evaluation factors

\begin{abstract}
For wireless sensor network (WSN), the first step is to deploy sensor nodes effectively into the target area. In fact, node deployment will greatly influence the lifetime and performance of WSN system. In this paper, we summarized the evolution of sensing models frequently used. Some main models' property is analyzed in details. The strategies are classified into three classes (static deployment, mobile deployment and hybrid deployment) based on the mobility of sensor nodes. Then, the typical algorithms and ideas are introduced and commented. Different performance evaluation factors for sensor network are also depicted. Finally, the developing direction of future research is discussed and anticipated.
\end{abstract}

\section{Introduction}

Node deployment is one of the key problems for designing and planning wireless sensor network (WSN) [1]. Reasonable deployment can optimize the space resources, reduce the cost and energy consumption and extend the lifetime of the whole network. Thus, the network can better fulfill such tasks as the environment perception, information access and data transmission.

Generally, the main factors which affect the node deployment are the number and location of the nodes in the target area. Proper number of WSN nodes should be used to implement the specific task. Accordingly, the key problems needed to be solved in WSN node deployment procedure consist of the coverage rate and connectivity, efficient deployment algorithm, and deployment quality evaluation. In this paper, we addressed these issues with detailed analysis of current research.

\section{Sensing Model of WSN Nodes}

In WSN's research field, we ever saw many sensing models.

0/1 model is also known as the disk model. It is frequently used in the theoretical derivation and mathematical proof. As for actual situation, 0/1 model is too simplistic and always shows large deviation.

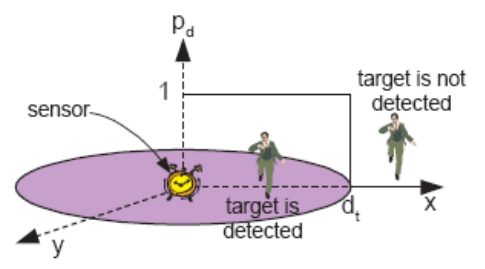

(a) 0/1 model

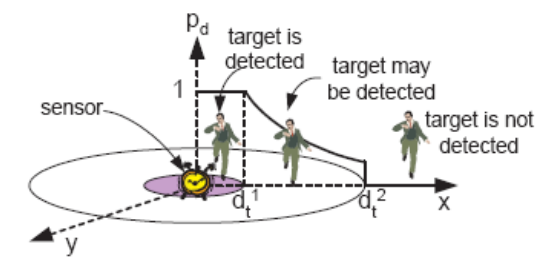

(b) Probability model

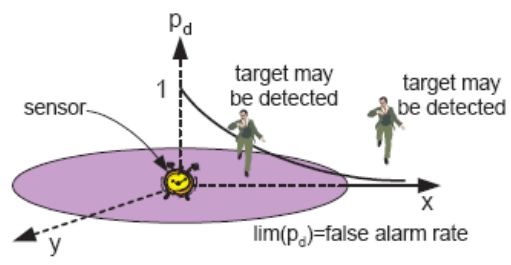

(c) False-alarm-rate model

Fig. 1 The detecting effect of different model

In fact, the node detects the target by measuring received signal strength, which will decrease instead of staying still while the distance increases. The probability for sensors to detect the target point perfectively will reduce exponentially while the Euclidean distance increases [2]. It is thought that the sensor cannot sense the target data correctly when signal strength is below some threshold.

0/1 model, coupled with the probability model, will divide the perception area into two areas at 
radial direction, with the circle inside as the entire perception area and the circle outside as the probability perception area. This constitutes the shadow model. When the radius of the inner circle becomes 0 , the model will reduce to the probability model. When the outside circle radius is equal to the inside circle, the model reduces to the disk model(0/1 model).

The common feature of $0 / 1$ and probability model is that they cover circular area. However, other sensors such as ultrasonic and vision own directional sensing ability at certain angle[4]. Therefore, the fan-shaped perception model is always used for visual sensor. Since it cannot depict directional sensors accurately, $\mathrm{Wu}$ [5] proposed a polygonal model with a series of vertices in polar coordinates, which can express the irregular boundary of the sensing area accurately. This model is more accurate than the fan-shaped model, but it is represented entirely on the geometric graph and too complicated for usage.

Differences among node devices and changes in environment will lead to different perception ability for the same type of sensor nodes. This will cause irregular sensing area for the nodes. He [6] proposed a concept, called DOI (degree of irregularity), to describe such irregularity. Defined as the maximal difference of wireless signal strength corresponding to sensing angle, DOI represents the change percentage of the maximal path loss during the transmission at unit direction. Along with the change of DOI, the perception and coverage area of the nodes becomes more and more irregular. When DOI is 0 , there will be no difference in signal strength, then the node model reduces to the ideal $0 / 1$ model.

Considering the influence of environment and nodes' components variance, irregular model can represents sensor nodes very well under actual situation. However, its application procedure is too complex and needs to test the environment actually. Another similar case is the approximate disk model [7].

The WSN node is consisted of electronics device and sensors which will inevitably produce misinformation and false alarm phenomenon. Researchers proposed a new sensing model, called false-alarm-rate model (FAR), based on Neumann- Pearson criterion [3]. Compared with the probability model, FAR model considers the unreliable factors of the node perception which is closer to the actual situation. In other words, FAR model will reduce to the probabilistic model by adjusting the parameters appropriately. Fig. 1 shows the relationship among 0/1 model, probability model and FAR model.

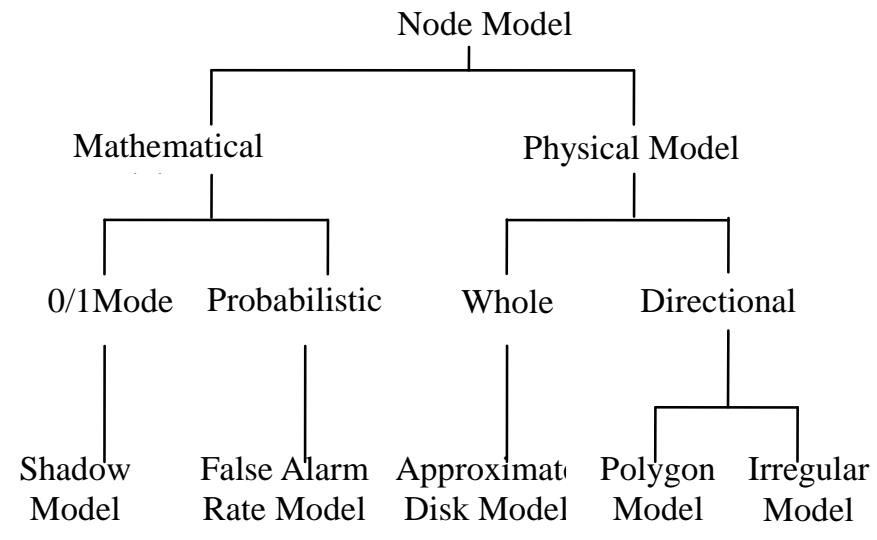

Fig.2 The evolution of the sensor node model

As a whole, although there are still some gaps, current node models are gradually approaching the true physical characteristics of the sensor. The relationship between different sensing models is summarized in Fig. 2.

\section{Senor Nodes Deployment Strategy}

According to nodes' mobility and placement controllability, WSN deployment can be divided into random deployment, deterministic deployment, etc. We can also give two different categories including homogeneous deployment and heterogeneous deployment on the basis of similarity and 
differences.

\section{Random Deployment.}

Random deployment generally means that the sensor nodes are thrown by aircraft randomly. For this operation, we can exert limited control on the nodes' distribution density[8,9].

Random deployment focuses on the scheduling strategy of the nodes through switching the working/sleeping state of the nodes to effectively monitor the target area. For instance, in order to control nodes' energy efficiently, there have appeared some algorithms such as nodes' working time scheduling scheme, distributed density control and distributed node- scheduling scheme. These schemes can effectively improve the network lifetime and performance to some extent.

Ishizuka [10] made deep research on the distribution function of randomly deployed nodes and proposed three deployment styles, including simple cast (two- dimensional normal distribution), uniform deployment and R-random deployment (nodes distributed in the radial direction of the base station uniformly).

\section{Deployment based on the rules.}

Some researchers think WSN deployment is a NP-complete optimization problem. Considering coverage rate, connectivity and life cycle, Molina [11] proposed a method called Proximity Avoidance Coverage-Preserving Operator which can enhance the optimization reliably and needs no additional calculation. As for homogenous sensor network, Yun [12] studies the deployment paradigm of full coverage and $k$ connectivity $(k \leq 6)$ with different communication/perception ratio. His conclusion is that there exists a common basic paradigm based on polygon which can be used to generate the best paradigm of all the known.

Since the target area is complicated and signal transmission is affected severely, indoor sensor network often adapt some rules-based deployment. The Gallery Alarm is a classic example, which builds a sensor network of full resolving power by coding theory with limited topology. But for actual application, the prerequisites of limited topology cannot be met. It is difficult to design algorithms to make some objective functions. As radio signals have serious diffraction and reflection in indoor propagation, it is difficult to build a unified model. Using the $2.4 \mathrm{GHz}$ ISM band and the logarithm distance model, Huo [13] proposes a rapid deployment program of WSN nodes while such factors as antenna height and other environmental are taken into account. By defining the deployment rules and supplementary rules, the author makes a beneficial exploration to avoid the perception holes caused by the antenna height variance.

Furthermore, deployment issues are researched by researchers from the viewpoint of gradient, nested grid and ring model respectively [14-16]. While [17] studies the directional WSN deployment.

\section{Mobile deployment.}

The nodes with mobility can build mobile sensor network with more reasonable deployment program.

Based on the artificial potential field, mobile node typically gets attractive and repulsive forces[18]. It expands the nodes gathering together to the surroundings by the repulsion among the nodes or the repulsion between the nodes and the barriers. As a result of this, network coverage is expanded. According to the composition of attractive force and repulsive force among the neighbor nodes, the barriers and the target, the cluster head node calculates the destination position of the other members in the cluster. The algorithms based on artificial potential field can make the nodes expand to the whole perception area quickly, but they do not consider the node crash which is able to appear, and they are easy to fall into the local optimal solution.

To overcome the limitations such as local minimum, the blockage of a narrow channel and vibration which will cause interference and instability to the system? Researchers have proposed many new methods. For example, Song [19] uses certainty degree to express the barriers abstractly, he divides the work area into many small square units, and each unit is associated with a certainty degree valued in $[0,1]$. Then, the network is forced by the virtual repulsive forces of the cells in the active windows to spread itself throughout the environment. There is also a deployment based on hierarchy potential field and the network coverage enhancement based on the virtual force [20], 
these algorithms enhance the application of the mobile sensor network deployment strategy in some degree.

Similar with the concept of the virtual force, Liao [21] proposes the node deployment algorithm based on firefly group optimization, the purpose is to enhance the network coverage of the initial random deployment. The algorithm regards each node as a distributed light of firefly, the strength depends on the distance between the sensor node and its neighbor node. The node is attracted by the nodes with low-intensity fluorescence and moves towards one of the nodes. Thus, the algorithm achieves maximum perception area by moving the nodes towards the low-perception strength area. As it is easy to result in coverage hole or network segmentation in spare network environment, there has been a self-deployment algorithm to face the spare deployment of the anti-division in mobile sensor network [22, 23].

At present, most algorithms are centralized, these algorithms assume that the node or the cluster head node can get the positions of the other nodes and determine where these nodes should move. In real situation, it is difficult to find a centralized server; we must adopt the efficient distributed algorithms. However, the research in this field is very rare at the moment.

Researchers have proposed some deployment strategy of limiting the moving range of the node because the energy consumption in node mobility is much larger than its perception and communication. The most limited mobility is a disposable mobility, that is to say, the node can only flip or jump once to the limited new position, so the goal is to achieve maximum coverage with minimum total hop. Because the optimized design is very difficult, Chellappan [24] proposes a strategy which describes the initial deployment of the network as a virtual figure. In his strategy, each network grid corresponds with a vertex in the figure, so he transforms the minimum hops and the maximum coverage into the minimum cost and the maximum flow in his figure, at last, we can use many algorithms to solve it. The disadvantage of the strategy is that it requires the length of the grid $\mathrm{d}$ and the perception radius of the node Rs meeting the constraint condition $d \leq R_{s} / \sqrt{2}$, which reduces the coverage of the nodes apparently. And it does not take the connectivity of the network into account.

In order to achieve the energy savings in the move of the node, it is necessary to establish the energy constraints and the optimized move according to the whole situation of the network [25-27]. Greco [26] studies the optimized deployment for nodes with all-direction perception but limited moving distance. His model supports complicated environment by two-step operation to find the local optima according to the discrete time gradient. The first step is to carry through rough optimization so that the node can be divided into clusters and move in planar area. Perception radius can also be changed to meet the simplified model of the environment. The node position achieved is mapped into the network for fine optimization of the second step. Through nodes' movement, the second step can be implemented by online distributed ways. Kuang [27] introduces the genetic algorithm which takes the jumping direction of the nodes as its gene. The fitness function which can achieve the optimal motion planning both considers the maximum coverage and the minimum moving distance. Fig. 3 shows the simulation result of mixed deployment with mobility- limited sensor nodes[25].

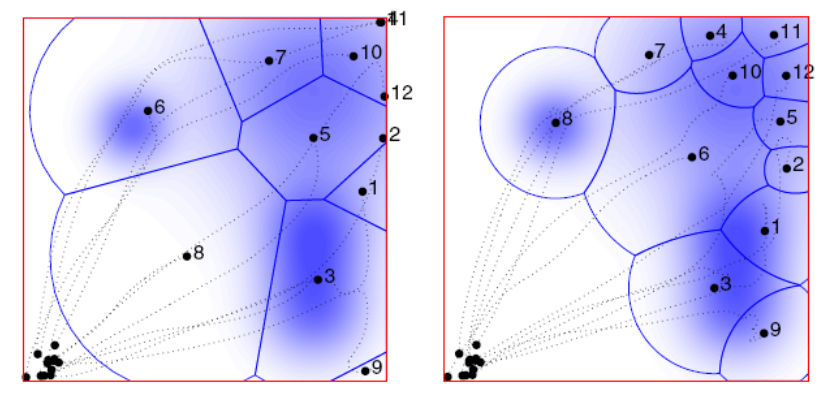

Fig.3 Simulation result of mixed deployment

There are some similar researches such as limited mobile deployment of grid point and probability or based on a neighbor of topological knowledge jump, etc. Since mobile deployment of multi-coverage network is relatively complicated, there is little research on it. A typical case is 
mobile sensor deployment optimization for k-coverage in wireless sensor networks with a limited mobility model[28]. It permits some nodes to move and fill the empty network. The strategy builds a disk model to limit the moving range of mobile nodes. Then particle swarm optimization is used to enhance the k-coverage of the network.

\section{Deployment of hybrid network.}

Adding suitable amount of heterogeneous nodes in WSN can improve the success rate of data transmission and extend the lifetime of the network.

For those heterogeneous node networks including mobile and fixed agent, Gani [29] divides WSN into perception layer, polymer layer and query layer. Among them, the mobile agent of the polymer receives data from the perception layer according to the principle of the nearest neighborhood. At the same time, Gani builds the utility function based on communication cost and controls mobile nodes to move from any initial position to the position where the utility value is minimum on the basis of gradient descent. For those anisotropic nodes, Akbarzadeh [30] thinks that current assumption of most deployment methods is too simple, so the results obtained are far away from the optimal value in actual situation.

Because it is difficult to build model for the environment, researchers mainly focus on energy consumption or the minimum cost, and fewer take the environment limitation and the factors of the route and energy into account. Introducing into mobile robot nodes to achieve flexible deployment of the wireless sensor network and its nodes, is especially important for the unknown environment.

For rapid response of the monitoring WSN, Xing [31] proposes a monitoring model which can make the static and the dynamic nodes collaborate effectively based on data fusion. By optimizing the scheduling, the model achieves the monitoring performance and reduces the cumulative distance of the nodes at the same time. Batalin [32] also thinks that the robot and the sensor network cooperate with each other in order to achieve coverage and exploration, so he proposes an efficient local optimization algorithm, that is the Least Recently Visited (LRV) algorithm, and proves that it is corresponding with the complete graph and optimal tree. Fig.4 shows the behavior switching for mobile sensor node. At different stage, the robot fulfills different task between SearchBeacon and AtBeacon[32].

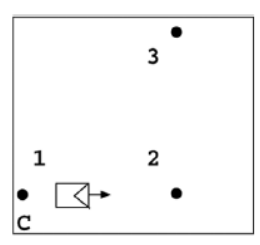

(a)

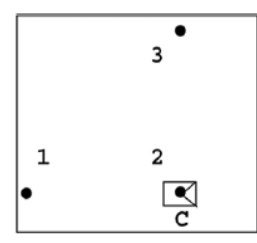

(b)

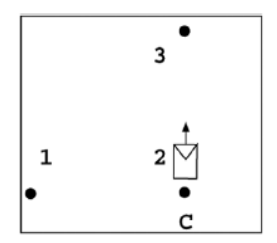

(c)

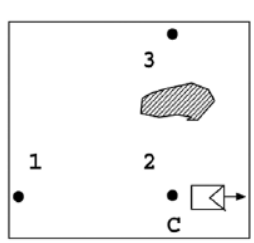

(d)

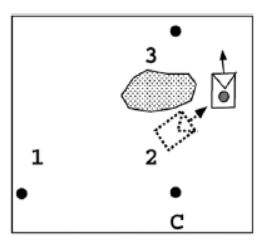

(e)

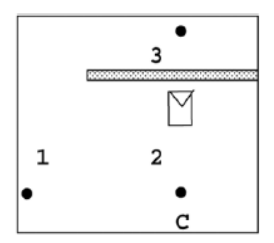

(f)

Fig.4 Behavior switching for mobile sensor node

Combining the aircraft and the sensor network to form a rapid deployment and a monitoring network of large-scale coverage, is a potential research, but the progress is slow because of the research conditions. At present, there are only a little well-known research on it. Corke [33] uses flying robot to deploy sensor network and to solve large-scale environmental monitor and emergency charges. Helicopter can communicate with the terrestrial network for network deployment, node location and network maintenance, and as a relay data transmission between disconnect networks. The system can realize the command control of the node deployment, and supports navigation, monitoring, search and rescue missions. The AWARE project [34] of the EU builds an experimental platform to provide the collaboration between the flying robot and ground-sensing of the implementation network. The project achieves self-deployment, self-configuring and self-healing of the sensor network by helicopter. At the same time, the project also builds a distributed, autonomous decision-making framework, and achieves the collaboration between the high bandwidth of the helicopter and the low bandwidth of the WSN by using middleware and publish/subscribe. 


\section{Evaluation of Sensor Network Deployment}

The evaluation of the network deployment is especially essential for analyzing and improving the strategy of the deployment, so researchers have proposed some indexes such as the covering strength, coverage rate and exposure, etc.

In sensor deployment area, the spatial distribution of network coverage intensity can be characterized by the field strength of the coverage. Assuming that there are $n$ sensors such as $s_{1}, s_{2}, \ldots, s_{n}$ on the working state in the area, if we regard $S\left(s_{i}, q\right)$ as the coverage quality of sensor $s_{i}$ at point $q$, then the field intensity of point $q$ can be expressed as:

$$
I(F, q)=\sum_{i=1}^{n} S\left(s_{i}, q\right)
$$

For the probability model, the field intensity of the coverage is often defined as joint detection probability of neighbouring nodes of point $q$, rather than simple addition. Assuming that the area is divided into $N$ grids and the joint detection probability required is $p_{\text {req }}$, then the field intensity of whole area is defined as:

$$
E=1-\frac{\sum_{i=1}^{N}\left|p_{i}-p_{\text {req }}\right| \times F_{\text {acum }}}{N \times p_{\text {req }}}
$$

The logical variable $F_{\text {acum }}$ is 1 when $P_{i}$ is less than $p_{\text {req }}$, otherwise it is 0 .

The detection area is sampled uniformly to extract $N$ effective grid points. Assuming that the number of the grid points is $k$ when the coverage field intensity arrives to the minimum detection probability, then the effective detection probability of the network can be defined as:

$$
p=\frac{\operatorname{GridNum}(k)}{N}
$$

Here, GridNum $(k)$ represents the degree that the $k$-th grid point meets the requirement of the detection probability. It is 1 when the degree is meted, otherwise it is 0 .

For the sensor network aiming at mobile target surveillance, exposure degree is often used to characterize the cumulative effect of the field intensity when the target goes through the surveillance area. Since the target's track can be any curve, it is difficult to give an accurate mathematical description. Therefore, Shen [35] redefines the exposure based on the grid division:

$$
E\left(p(i), p_{e}, p_{s}\right)=\sum_{i=1}^{i_{\max }} I(F, \operatorname{Grid}(i))|p(i)|
$$

In the formula (4), $|p(i)|$ is the length of the mobile track $p(i)$. At the same time, $i$ is the grid number while $i_{\max }$ is the total number. If we regard the $i$-th grid that the track goes through as Grid(i), then the corresponding field intensity is I(F,Grid(i)).

\section{Summary}

For the optimized deployment of wireless sensor network, many scientific issues must be considered combining with technological and engineering factors. There are still many challengeable problems to be solved. Here we can list some topic of future research:

(1) The perception models and algorithms close to the actual physical situations. Most of the existent perception models do not consider communication interference and the direction of sensor nodes. There exists a large deviation from real situation. Meanwhile, the perception area is usually of irregular shapes. Background noise should also be considered.

(2) Fusing the application background and the field knowledge together. WSN is an application-related network. It is necessary to combine the environment factors with expert knowledge so that optimal deployment can be obtained. In addition, it is also easy to implement through context-sensitive, application-oriented and information localization to improve the algorithms. 
(3) Deployment of the WSN network in three- dimensional space. With the development of WSN in underwater and underground environment, the importance of the three-dimensional network has emerged gradually. But most of the deployment algorithms in two-dimensional space will evolve into NP-Hard problem in three-dimensional pace[36]. Therefore, we must propose new strategies.

(4) WSN Network Re-deployment and maintenance. With time elapsing, the network topology will be changed due to nodes' hardware failure or energy depletion[37]. Thus it is necessary to make re-deployment and maintainance.

(5) Hybrid deployment of different kinds of nodes. Deployment of heterogeneous nodes is seldom investigated at present. It is necessary to strengthen the research of the large-scale heterogeneous deployment for different nodes with variable functions and types.

(6) WSN middleware. Middleware [38] will be beneficial for the rapid deployment of WSN. It can hide the data source in virtual abstraction layer of the sensor so as to build simple, unified API port and interact with the sensor network.

\section{References}

[1] Jason H. Li, Miao Yu, Sensor Coverage in Wireless Ad Hoc Sensor Networks[J]. International Journal of Sensor Networks, 2007, 2(3-4): 218-229.

[2] Yi Zou, Krishnendu Chakrabarty. Sensor Deployment and Target Localization in Distributed Sensor Networks[J]. ACM Transactions on Embedded Computing Systems, 2004, 3(1): 61-91.

[3] Yang Yang, Rick S. Blum. Routing for Emitter/Reflector Signal Detection in Wireless Sensor Network Systems[C]. IEEE Int Conf on Communications(ICC '07), Jun. 2007: 4919-4924

[4] Huadong Ma, Yonghe Liu. On Coverage Problems of Directional Sensor Networks [M]//Mobile Ad-hoc and Sensor Networks. Springer Berlin Heidelberg, 2005: 721-731.

[5] Chun-Hsien Wu, Yeh-Ching Chung. A Polygon Model for Wireless Sensor Network Deployment with Directional Sensing Areas[J]. Sensors, 2009, 9(12): 9998-10022.

[6] He T., Huang C., Blum B.M. Range-free Localization and Its Impact on Large Scale Sensor Networks[J]. ACM Transactions on Embedded Computing Systems (TECS), 2005, 4(4): 877-906.

[7] Kuhn F., Wattenhofer R., Zollinger A. Ad Hoc Networks Beyond Unit Disk Graphs[J]. Wireless Network, 2008, 14(5): 715-729.

[8] J. Tang, B. Hao, A. Sen. Relay Node Placement in Large Scale Wireless Sensor Network[J]. Computer communications, 2006, 29(4): 490-501.

[9] D. Tian, N. D. Georganas. A Node Scheduling Scheme for Energy Conservation in Large Wireless Sensor Networks [J]. Wireless Communications and Mobile Computing, 2003, 3(2): 271-290.

[10]M. Ishizuka, M. Aida. Performance Study of Node Placement in Sensor Networks[C]. 24th International Conference on Distributed Computing Systems Workshops, IEEE, 2004: 598-603.

[11]Guillermo Molina, Francisco Luna, Antonio J. Nebro, Enrique Alba. An Efficient Local Improvement Operator for The Multi-objective Wireless Sensor Network Deployment Problem[J]. Engineering Optimization, 2011, 43(10): 1115-1139.

[12]Ziqiu Yun, Xiaole Bai, Dong Xuan, Lai T.H., Weijia Jia. Optimal Deployment Patterns for Full Coverage and k-Connectivity Wireless Sensor Networks[J]. IEEE/ACM Transactions on Networking, 2010, 18(3): 934-947.

[13]Huo Hongwei, Gao Shuai, Niu Yanchao,Zhang Sidong. A deployment strategy for wireless sensor networks using indoor radio propagation model[J]. Engineering Science, 2008, 10(9): 64-69. 
[14]Wen Jun, Jian Qiang, Jiang Jie, Dou Wenhua. A Coverage-Guaranteed Gradient Nodes Placement for Wireless Sensor Networks [J]. Computer Engineering \& Science, 2008, 30(6): 86-91.

[15] Aitsaadi N., Achir N., Boussetta K., Gavish B. A Gradient Approach for Differentiated Wireless Sensor Network Deployment[C]. Wireless Days 2008(WD '08), 1st IFIP, Dubai, Nov. 2008: 1-5.

[16]Liu Liping, Cao Feng, Wang Zhi, Sun You Xian. A Nested -Grid-Based Energy-efficient Deployment Algorithm for Wireless Sensor Networks [J]. Information and Control, 2006, 35(2):154-160.

[17]Han X., Cao X., Loyd E.L., Shen C.C. Deploying Directional Sensor Networks with Guaranteed Connectivity and Coverage[C]. 5th Annual IEEE Communications Society Conference on Sensor, Mesh and Ad Hoc Communications and Networks, San Francisco, CA, USA, June, 2008: 153-160.

[18]A. Howard, M. J. Mataric, G. S. Sukhatme. Mobile Sensor Networks Deployment using Potential Fields: A Distributed, Scalable Solution to the Area Coverage Problem[C]. 6th International Symposium on Distributed Autonomous Robotics Systems, Japan, June, 2002.

[19]Song Guangming, Zhuang Wei, Wei Zhigang, Song Aiguo. Self-Deployment Algorithm for Mobile Sensor Networks in Unknown Environment[J]. Journal of South China University of Technology, 2006, 34(9): 26-30.

[20]Lee J, Dharne A D, Jayasuriya S. Potential Field Based Hierarchical Structure for Mobile Sensor Network Deployment[C]. 2007 American Control Conference, New York, 2007, 5946-5951.

[21]Wen-Hwa Liao, Yucheng Kaoa,Ying-Shan Li. A Sensor Deployment Approach Using Glowworm Swarm Optimization Algorithm in Wireless Sensor Networks[J]. Expert Systems with Applications, 2011, 38(10): 12180- 12188.

[22] Yang Minghua, Tan Li, Cao Yuanda, Yu Jiong. Anti-Partition Self-Deployment Algorithm in Mobile Sensor Network [J]. Journal of Beijing University of Posts and Telecommunications, 2009, 32(4): 109-112.

[23]D. Lymberopoulos, A. Savvides. XYZ: A Motion-enabled, Power Aware Sensor Node Platform for Distributed Sensor Network Applications[C]. International Symposium of Information Process Sensor Network (IPSN), Los Angeles, CA, Apr. 2005: 449-454.

[24]Chellappan S., Bai X., Ma B., et al. Mobility Limited Flip-Based Sensor Network Deployment [J]. IEEE Trans on Parallel and Distributed Systems, 2007, 18(2): 199-211.

[25]Andrew Kwok, Sonia Martinez. Deployment Algorithms for A Power-constrained Mobile Sensor Network[J]. International Journal of Robust and Nonlinear Control, 2010, 20(7): 745-763.

[26]Greco L., Gaeta M., Piccoli B. Sensor Deployment for Network-Like Environments[J]. IEEE Transactions on Automatic Control, 2010, 55(11): 2580-2585.

[27]Kuang Linai, Cai Zixing. Genetic algorithm based redeployment scheme in wireless sensor networks [J]. Control and Decisio, 2010, 25(9): 1329-1332.

[28]Xingzhen Bai, Shu Li, Juan Xu. Mobile Sensor Deployment Optimization for k-Coverage in Wireless Sensor Networks with a Limited Mobility Model[J]. IETE Technical Review, 2010, 27(2): 124-137.

[29]Gani M. Optimal Deployment Control for A Heterogeneous Mobile Sensor Network[C]. 9th International Conference on Control, Automation, Robotics and Vision, Dec. 2006: 1-6.

[30]Vahab Akbarzadeh, Albert Hung-Ren Ko, Christian Gagne, Marc Parizeau. Topography-Aware Sensor Deployment Optimization with CMA-ES[M]//Parallel Problem Solving from Nature, 
Springer Berlin Heidelberg, 2010: 141-150.

[31]Guoliang Xing, Jianping Wang, Zhaohui Yuan, et al. Mobile Scheduling for Spatiotemporal Detection in Wireless Sensor Networks[J]. IEEE Transactions on Parallel and Distributed Systems, 2010, 21(12): 1851 -1866.

[32]Batalin M. A., Sukhatme G. S. The Design and Analysis of An Efficient Local Algorithm for Coverage and Exploration Based on Sensor Network Deployment[J]. Robotics, 2007, 23(4): 661-675.

[33]Corke P., Hrabar S., Peterson R., et al. Autonomous Deployment and Repair of a Sensor Network Using an Unmanned Aerial Vehicle[C]. IEEE International Conference on Robotics and Automation, 2004, 4: 3602-3608.

[34]Ollero A., Kondak K., Previnaire E., etc. Integration of Aerial Robots and Wireless Sensor and Actuator Networks: The AWARE project[C]. IEEE International Conference on Robotics and Automation, 2010: 1104 -1105.

[35] Jia Jie, Chen Jian, Chang Guiran, Zhao Linliang. Optimal Coverage Algorithm of Sensor Nodes Set Selection in Wireless Sensor Network [J]. Journal of Northeastern University, 2007, 28(11): 1560-1563.

[36]Dario Pompili, Tommaso Melodia, Ian F. Akyildiz. Three-dimensional and Two-dimensional Deployment Analysis for Underwater Acoustic Sensor Networks[J]. Ad Hoc Networks, 2009, 7(4): 778-790.

[37]Renjie Huang, Wen-Zhan Song, Mingsen Xu, Nina Peterson, et al. Real-World Sensor Network for Long-Term Volcano Monitoring: Design and Findings[J]. IEEE Transactions on Parallel and Distributed Systems, 2012, 23(2): 321-329.

[38]Wouter Horré, Sam Michiels, Wouter Joosen, Danny Hughes. Advanced Sensor Network Software Deployment using Application-level Quality Goals[J]. Journal of Software, 2011, 6(4): 528-535 